EPJ Web of Conferences 47, 13001 (2013)

DOI: $10.1051 /$ epjconf/20134713001

(C) Owned by the authors, published by EDP Sciences, 2013

\title{
High-precision ground-based photometry of exoplanets
}

\author{
Ernst J.W. de Mooija and Ray Jayawardhana \\ Department of Astronomy and Astrophysics, University of Toronto, 50 St. George street, \\ Toronto ON, M5S 3H4, Canada
}

\begin{abstract}
High-precision photometry of transiting exoplanet systems has contributed significantly to our understanding of the properties of their atmospheres.

The best targets are the bright exoplanet systems, for which the high number of photons allow very high signal-to-noise ratios. Most of the current instruments are not optimised for these high-precision measurements, either they have a large read-out overhead to reduce the readnoise and/or their field-ofview is limited, preventing simultaneous observations of both the target and a reference star. Recently we have proposed a new wide-field imager for the Observatoir de Mont-Megantic optimised for these bright systems (PI: Jayawardhana). The instruments has a dual beam design and a field-of-view of 17' by 17'. The cameras have a read-out time of 2 seconds, significantly reducing read-out overheads.

Over the past years we have obtained significant experience with how to reach the high precision required for the characterisation of exoplanet atmospheres. Based on our experience we provide the following advice:

1. Get the best calibrations possible. In the case of bad weather, characterise the instrument (e.g. nonlinearity, dome flats, bias level), this is vital for better understanding of the science data.

2. Observe the target for as long as possible, the out-of-transit baseline is as important as the transit/eclipse itself. A short baseline can lead to improperly corrected systematic and mis-estimation of the red-noise.

3. Keep everything (e.g. position on detector, exposure time) as stable as possible.

4. Take care that the defocus is not too strong. For a large defocus, the contribution of the total flux from the sky-background in the aperture could well exceed that of the target, resulting in very strict requirements on the precision at which the background is measured.
\end{abstract}

\section{INTRODUCTION}

High precision ground-based observations of exoplanets have played an important role in the characterisation their atmospheres. Both during transit [e.g. 1, 2] as well as during the secondary eclipse [e.g. 3-5] and even for non-transiting planets [6, 7].

All these observations require very high precision in order to detect the small signals induced by the atmosphere ( $\lesssim 3$ mmag for secondary eclipses in the near-infrared and a few times $10^{-4}$ for transmission spectroscopy). In Section 2 we present some lessons learned from our observations of exoplanets from the GROUnd-based Secondary Eclipse (GROUSE) project [8] and from related observations and in Section 3 we present a new instrument for the characterisation of the brightest transiting exoplanet systems.

\section{LESSONS LEARNED FROM HIGH-PRECISION PHOTOMETRY}

During the past few years we have been using ground-based telescope to study exoplanets. Here we present some of the lessons we learned and provide some suggestions for future observations.

\footnotetext{
ae-mail: demooij@astro.utoronto.ca
}

This is an Open Access article distributed under the terms of the Creative Commons Attribution License 2.0, which permits unrestricted use, distribution, and reproduction in any medium, provided the original work is properly cited. 

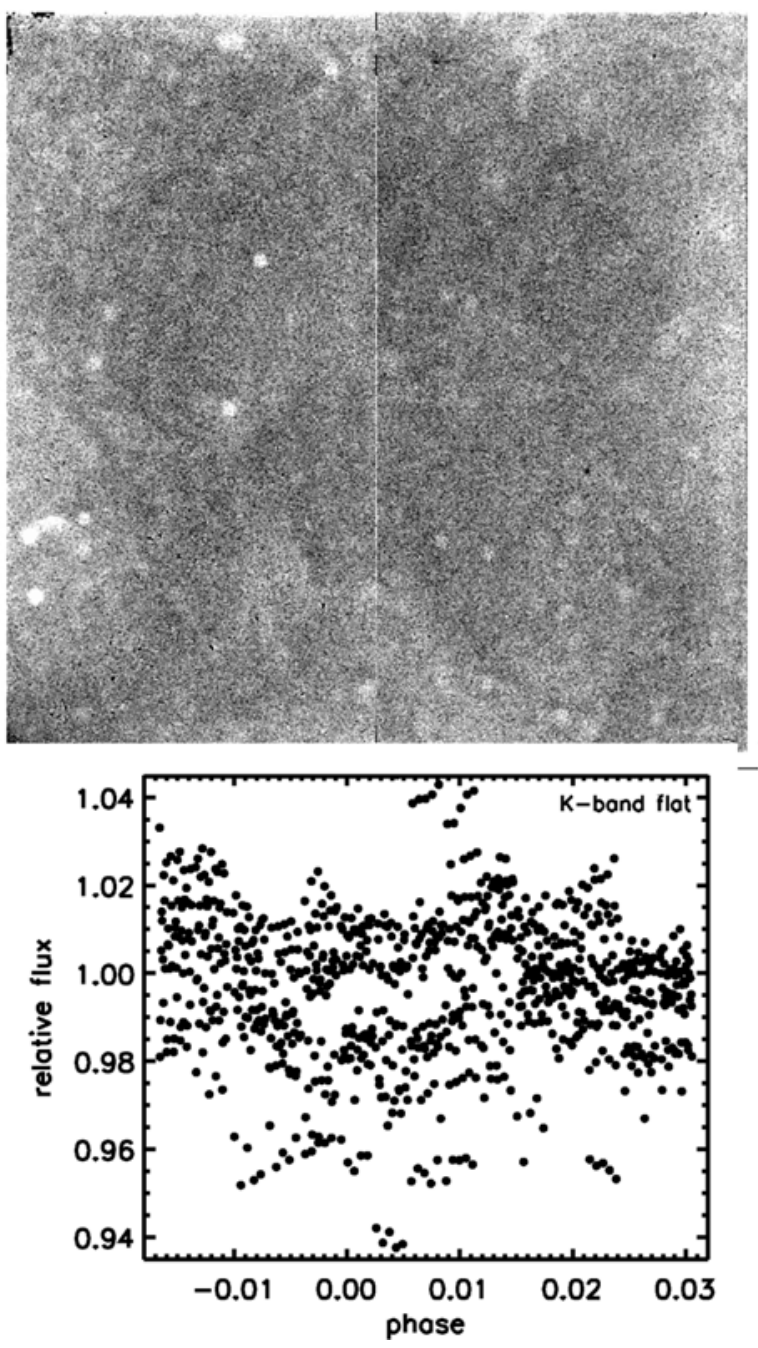

Figure 1. A transit of HAT-P-1b in K-band (bottom panel) for data flatfielded with the K-band flatfield containing dust glow (top panel).

\subsection{Get good calibrations}

A good understanding of the instrument used is very important. Obtaining the best possible calibration frames is therefore necessary. In some cases it might even be better to use no calibration frames at all rather than bad calibration frames. In 2008 a transit of HAT-P-1b was observed in the K-band using the UKIRT. After the observations only night-time sky-flats were obtained. In the K-band dust on the dewar window glows, resulting in bright spots in both the image and the flat-field (Fig. 1, top panel). Since these dust-spots are an additive effect and do not correspond to any real sensitivity map of the detector, using this flatfield creates strong systematic effects, especially when the target is moved across the detector (Fig. 1, bottom panel). However, using J-band flat field obtained during the same night (Fig. 2, top panel) the transit becomes clearly visible (Fig. 2, bottom panel). 

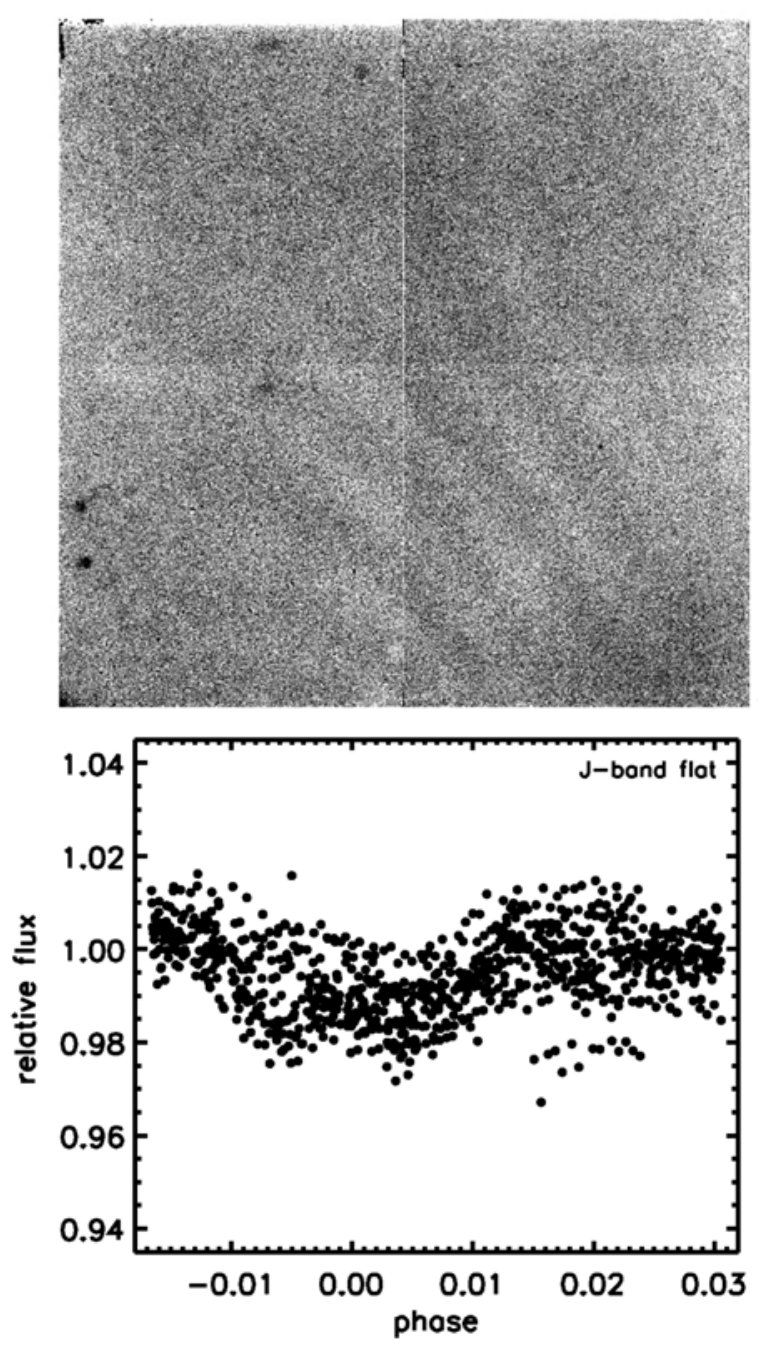

Figure 2. Same data as used for Fig. 1, but now flatfielded with a J-band flatfield that does not contain bright dust spots (top panel).

\subsection{Keep everything as stable as possible}

With the secondary eclipse observations of TrES-3b [3], the observations were dithered, in order to subtract the background. For the $\mathrm{K}_{s}$-band observations of HAT-P-1b with LIRIS [9] the strategy was changed to staring mode, where the stars were kept on the same region of the detector. This has two advantages:

1. The overheads were significantly reduced because no telescope offsets needed to be performed. This in turn enabled the use of long sequences frames which in the case of the LIRIS instrument on the William Hershel Telescope (WHT), enabled the exclusion of frames affected by the reset anomaly (a strong ramp in the background that the first 1-5 frames of a sequence suffer from).

2. Since the star was kept within a few pixels of its original position, the impact of flatfielding errors was significantly reduced, increasing the precision. 


\section{EPJ Web of Conferences}

\subsection{Observe the target for as long as feasible}

For high-precision photometry it is necessary to obtain as large an out-of-transit baseline as possible, since this baseline provides the level with respect to which the transit/eclipse depth is measured and is also used to correct for systematic effects in the data.

As part of the GROUSE project, a secondary eclipse of WASP-33b was observed in the $\mathrm{K}_{s}$-band on two different nights in 2010 [10]. On the first night unfortunately only a short out-of-eclipse baseline was observed. Therefore, when simultaneously fitting for the eclipse and the systematic effects, there is a strong degeneracy between the eclipse depth and the baseline parameters, especially when using a polynomial baseline model. For the second night, where the baseline was significantly longer the degeneracies are much weaker.

\subsection{Too strong defocus can increase the noise}

Defocusing the telescope is a strategy used for most observations. By defocusing the light of the target is spread over many pixels, reducing the impact of pixel-to-pixel variations and allowing longer exposure times without saturations by reducing the peak flux. However, when defocusing, the radius of the aperture used for aperture photometry needs to be increased in order to include all the flux from the source. This also increases the contribution of the sky-background to the total flux in the aperture. The increased noise from the sky-background reduces the signal-to-noise ratio, although the impact in the scintillation dominated regime (e.g. for bright stars, short exposure times) is relatively small.

However, a bigger problem is the accurate removal of the background flux. Typically the background is determined in an annulus around the target, scaled to the correct number of pixels and then subtracted. As the relative contribution of the background to the total flux in the aperture increases, the precision at which the background needs to be known also increases. If the sky contributes as much flux as the target the sky needs to be determined at the same (or better) precision as the target in order to avoid introducing extra noise. Uncorrected effects such as a small residual gradient in the sky-background and hot-pixels could potentially limit the precision that can be reached. This effect is worse in the near-infrared, where the sky-brightness is higher than in the optical. In Fig. 3 we show this relative contribution as a function of aperture size for a bright target in the $\mathrm{V}$-band $\left(V=8.0\right.$, top panel) and $\mathrm{K}_{s}$-band $(K=5.5$, bottom panel). As can be seen, for the V-band the sky-background starts to contribute significantly to the total flux only for the largest apertures, however, in the $\mathrm{K}_{s}$-band, apertures of $\sim 10$ " or more have a higher contribution from the sky-background then from the target.

\section{A WIDE-FIELD IMAGER FOR THE CHARACTERISATION OF BRIGHT TRANSITING EXOPLANETS SYSTEMS}

Bright transiting planets are ideal for studying their atmospheric properties. The many photons received from the system allow very high signal to noise ratios to be reached ( $>4000$ per minute). However, the large number of photons also poses a problem, since they far exceed the saturation limit of the detector. To avoid this problem, instruments are typically defocused (e.g. De Mooij \& Snellen 2009), and short exposure times are used. Most imagers that are currently available have read-out overheads of tens of seconds, making for inefficient observations. In Fig. 4 we show the overheads as a function of exposure times for different detector read-out times. For a typical instrument with a $30 \mathrm{sec}$ overhead, the efficiency exceeds $50 \%$ only for exposure times longer than 30 seconds. Increasing the exposure times can be achieved by increasing the defocus, which can also lead to problems (see Sec. 2.4).

In addition, high-precision ground-based photometry requires reference stars to correct for atmospheric and instrumental effects. These reference stars should be of similar brightness to the target in order to avoid reducing the signal-to-noise ratio. For the brightest transiting exoplanet systems 


\section{Hot Planets and Cool Stars}
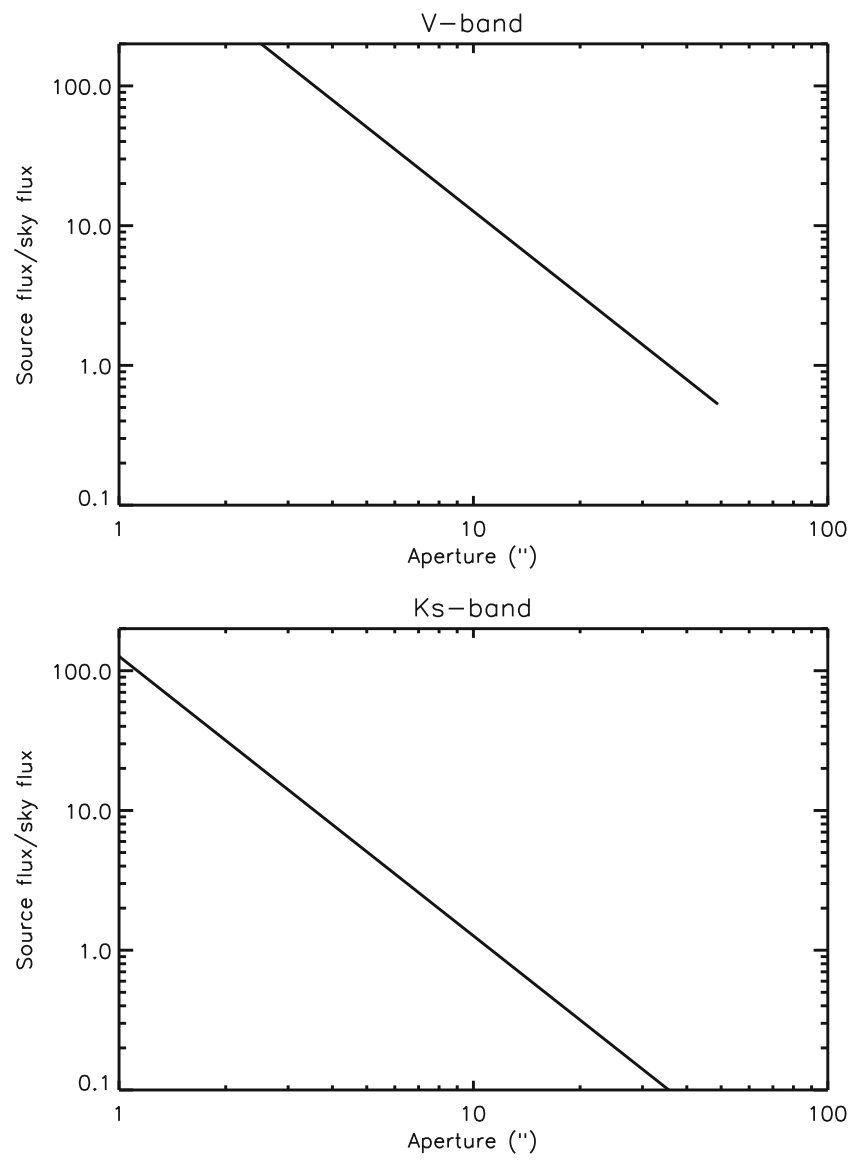

Figure 3. Relative contribution of the sky-background to the flux from the target as a function of aperture for a $V=8.0$ star during bright time (top panel) and a $K=5.5 \mathrm{star}$ (bottom panel). As the relative flux from the sky-background increases, so does the requirement on the precision at which the sky-background is determined.

$(\mathrm{V} \lesssim 9$ mag) the average separation between the target and reference stars becomes large, requiring instruments with a large field of view.

We have recently submitted a proposal (PI: Jayawardhana) for a wide-field imager with a fast read-out in order to characterise the atmospheres of the brightest systems with a transiting planet. The instrument will be mounted at the Cassegrain focus of the 1.6 meter telescope at the Observatoir du Mont-Megantic (OMM), but it can be adapted to work with other telescopes. In order to further increase the efficiency the instrument uses a beamsplitter enabling observations at two wavelengths simultaneously. Off-the-shelf cameras are used to decrease the cost and complexity. The current design of the instrument uses two cameras, each with a back-illuminated EEV230 CCD with $15 \mu$ m pixels in a 2048 by 2048 pixel array. The camera has a read-out overhead of 2 seconds, allowing for $>80 \%$ duty cycle for exposure times over 8 seconds (e.g. Fig. 4). In addition, each of the cameras is equipped with a filterwheel and a focuser, which allows the focus to be adjusted for each channel individually, compensating for the brightness difference of the star in the red and blue arm. Since the intrinsic pixelscale for $15 \mu \mathrm{m}$ pixels at the OMM is $0.24^{\prime \prime}$, resulting in a field-of-view of only $8.2^{\prime}$ by $8.2^{\prime}$, a focal reducer is used to increase the field-of-view. The current design is based on that of the Panoramix-II instrument at the OMM [11] and has been adapted to the size of the proposed cameras, giving a FOV of $17^{\prime}$ by $17^{\prime}$, and for dual beam operations. 


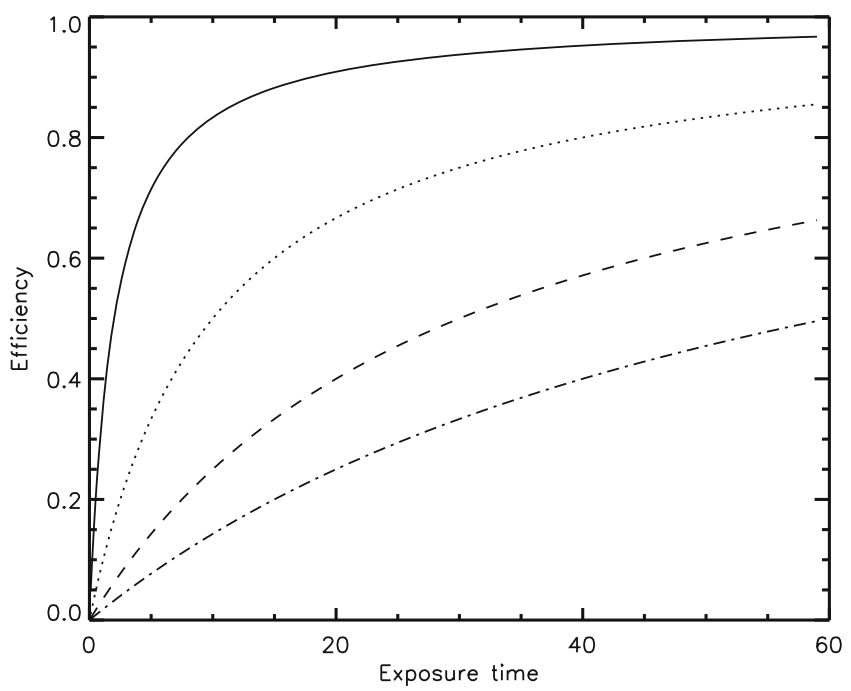

Figure 4. The on-sky integration efficiency as a function of the exposure time for different read-out overheads. The overheads are $2,10,30$ and 60 seconds respectively from top to bottom.

In addition to characterising the atmospheres of the brightest exoplanet systems, the instrument will also be used to improve the strategies used for high-precision photometry (e.g. optimising the defocus and the exposure times).

\section{References}

[1] I.A.G. Snellen, S. Albrecht, E.J.W. de Mooij, R.S. Le Poole, A\&A 487, 357 (2008), 0805.0789

[2] M. Brogi, I.A.G. Snellen, R.J. de Kok, S. Albrecht, J. Birkby, E.J.W. de Mooij, Nature 486, 502 (2012), 1206.6109

[3] E.J.W. de Mooij, I.A.G. Snellen, A\&A 493, L35 (2009), 0901.1878

[4] D.K. Sing, M. López-Morales, A\&A 493, L31 (2009), 0901.1876

[5] B. Croll, L. Albert, D. Lafrenière, R. Jayawardhana, J.J. Fortney, ApJ 717, 1084 (2010), 1005.3027

[6] I.A.G. Snellen, R.J. de Kok, E.J.W. de Mooij, S. Albrecht, Nature 465, 1049 (2010), 1006.4364

[7] F. Rodler, M. Lopez-Morales, I. Ribas, ApJ1 753, L25 (2012), 1206.6197

[8] E. de Mooij, R. de Kok, B. Nefs, M. Brogi, I. Snellen, The GROUnd-based Secondary Eclipse project - GROUSE, in IAU Symposium, edited by A. Sozzetti, M.G. Lattanzi, A.P. Boss (2011), Vol. 276 of IAU Symposium, pp. 487-488

[9] E.J.W. de Mooij, R.J. de Kok, S.V. Nefs, I.A.G. Snellen, A\&A 528, A49+ (2011), 1103.0035

[10] E.J.W. de Mooij, M. Brogi, R.J. de Kok, I.A.G. Snellen, M.A. Kenworthy, R. Karjalainen, ArXiv e-prints (2013), 1301.3380

[11] S. Thibault, M. Wang, P. Côté, L. Drissen, É. Brière, Optics integration of the OMM wide-field visible camera (Panoramix-II), in Society of Photo-Optical Instrumentation Engineers (SPIE) Conference Series (2006), Vol. 6269 of Society of Photo-Optical Instrumentation Engineers (SPIE) Conference Series 